\title{
Modelos de series temporales aplicados a la predicción del tráfico aeroportuario español de pasajeros: Un enfoque agregado y desagregado*
}

\author{
ANA M. LÓPEZ ${ }^{a}$, MARIO A. FLORES ${ }^{b}$, JUAN I. SÁNCHEZ ${ }^{c}$ \\ a Instituto L.R. Klein, Universidad Autónoma de Madrid, Facultad de CC.EE. y EE., c/ Francisco \\ Tomás y Valiente , 5, 28049 Madrid, España.E-mail: ana.lopez@uam.es \\ b INECO, Paseo de la Habana, 138, 28036 Madrid, España. E-mail: mario.flores@ineco.com \\ c Universidad Autónoma de Madrid, Facultad de CC.EE. y EE., c/ Francisco Tomás y Valiente , 5, \\ 28049 Madrid, España. E-mail: juanignacio.sanchez@uam.es
}

\begin{abstract}
RESUMEN
La utilidad de las predicciones económicas es un hecho constatado en los países desarrollados para anticiparse a la toma de decisiones o a la aplicación concreta de políticas económicas. El tráfico aéreo de pasajeros es una de las principales magnitudes de actividad económica de un país, especialmente relevante en España, destino turístico internacional de primera magnitud. En este trabajo abordamos la realización de predicciones del tráfico total de pasajeros aéreos a nivel nacional a partir de la evolución mensual disponible en AENA, con modelos SARIMAX. Examinamos si, y en qué medida, el poder predictivo del tráfico aeroportuario de pasajeros en España, en términos agregados, puede ser mejorado mediante el uso de información más detallada e individualizada, a través de un enfoque desagregado (procedente de la suma de las predicciones realizadas para los diferentes aeropuertos regionales), en términos de la fiabilidad y precisión de los resultados.
\end{abstract}

Palabras clave: Predicción económica, SARIMAX, tráfico aéreo pasajeros.

\section{Forecasting of Spanish Passenger Air Traffic Based on Time Series Models with Aggregated and Disaggregated Approaches}

\begin{abstract}
The usefulness of economic forecasts is a fact in developed countries in order to anticipate the decision-making process or the specific implementation of economic policies. Air passenger traffic is one of the main economic magnitudes in a country, especially relevant in Spain, one of the worldwide tourist destination leaders. In this paper, it is addressed the forecasting of total national air passenger traffics, according to the monthly evolution registered by AENA, using SARIMAX models. It is tested whether, and to what extent, the predictive power of aggregated air passenger traffic in Spain can be improved by using more detailed and individualized information through a disaggregated approach (from the addiction of the individual regional airports forecasts), in terms of reliability and accuracy of results.
\end{abstract}

Keywords: Economic Forecasting, SARIMAX, Passenger Air Traffic.

Clasificación JEL: C53, L93

\footnotetext{
* Los autores agradecen los comentarios y sugerencias de dos evaluadores anónimos que han contribuido a la mejora de la versión final del trabajo que aquí se presenta. Las opiniones reflejadas no coinciden necesariamente con INECO.
}

Artículo recibido en febrero de 2017 y aceptado en mayo de 2017

Artículo disponible en versión electrónica en la página www.revista-eea.net, ref. ə-35211 


\section{INTRODUCCIÓN}

En el marco de este volumen dedicado a la "Predicción Económica: métodos y herramientas", presentamos un modelo de predicción de las series temporales de tráfico aeroportuario de pasajeros en España, desde la perspectiva de la dualidad del enfoque agregado frente al desagregado. En primer lugar, nos referiremos al marco general del proceso de predicción en economía para particularizar en el objeto de desarrollo del trabajo.

\subsection{Consideraciones sobre la predicción aplicada a la economía}

El interés por la predicción económica y empresarial se ha visto favorecido desde mediados del siglo pasado, plausible en las numerosas publicaciones científicas al respecto, además del reconocimiento de la academia. Hay un amplio consenso respecto a la utilidad de las predicciones económicas, hecho constatado en los países desarrollados. Como señala Graefe (2015), las personas, empresas y organizaciones habitualmente toman decisiones importantes, combinando la información de múltiples entradas y, en ese proceso, la predicción siempre es útil. Esta utilidad de la predicción se ve acrecentada si se optimizan los recursos empleados y si, además, se consigue acercar el resultado al usuario final. Para Green y Armstrong (2015), es aconsejable y conveniente sopesar que los procedimientos técnicos utilizados para la predicción económica deberían ser siempre lo bastante simples (sencillos de interpretar) como para ser entendidos por cualquier usuario de las predicciones.

Con la aplicación de diferentes técnicas y modelos a la información cuantitativa disponible, los economistas obtienen predicciones de un futuro cercano, pero incierto, con la finalidad de poder anticiparse al devenir de los acontecimientos y mejorar la toma de decisiones (López, 2016b). No obstante, el proceso de predicción económica no es una tarea sencilla, como puntualiza Kleinhenz (2016): "La verdad es que el pronóstico económico no es fácil. Mientras tratemos de ser tan cuidadosos, serios y científicos como sea posible, es una mezcla de arte y ciencia. El hecho es que el curso futuro de la economía es incierto, y nadie puede predecir con un alto grado de exactitud cómo sucederán las cosas”. En la misma línea insiste Pulido (2006) "Nadie debiera confundir la necesidad de dedicar tiempo y esfuerzo en pensar en términos de futuro, con realizar o comprar predicciones con técnicas más o menos sofisticadas. Navegar por el futuro exige imaginación, experiencia, información selectiva, incluso un grado razonable de intuición. Con muchos datos y estudios no se suplen estas carencias”. Velasco (2016) incide en la importancia de la predicción económica y su carácter multidisciplinar: "quiérase o no, la predicción económica forma parte del conocimiento científico y arrastra tras de sí una amplia tarea de investigación de economistas, estadísticos, matemáticos y expertos en múltiples campos de las ciencias sociales”. 
El proceso de predicción económica se basa en la moderna metodología econométrica que, tal y como la conocemos en el presente, comenzó a principios de los años 40, e incorpora tanto los desarrollos en el tratamiento de series temporales como la econometría espacial, siendo necesario el empleo de técnicas/modelos estadístico-econométricos para alcanzar un nivel suficiente de precisión (Pulido y López, 1999; López, 2016a). Una teoría general de predicción económica requiere una formalización matemática para clarificar por qué y cómo los aspectos importantes de la predicción empírica resultan de características específicas de la implementación de los pronósticos Ericsson (2016).

Para elegir un modelo/técnica de predicción satisfactorio pueden considerarse las indicaciones de Hendry y Richard (1983): los modelos deben ser consistentes con la teoría subyacente, coherentes con los datos y deben abarcar una amplia gama de modelos rivales. Pulido (2006) detalla que un sistema complejo de predicción económica combina diversos ámbitos (internacional, nacional, regional y sectorial), con distintas periodicidades (anuales, trimestrales y mensuales), diferentes técnicas (opiniones de expertos, series temporales, modelos) y fuentes externas de predicción o incluso redes. Armstrong (2001) persevera en la evaluación de predicciones alternativas, que señala como una tarea compleja pero ineludible. En conjunto, el esfuerzo de predicción siempre merecerá la pena si se tienen en consideración los riesgos implícitos en el ejercicio de la predicción y la interpretación correcta de los resultados. Pulido (2006) matiza: “aún con todos sus errores potenciales, la predicción supone una referencia imprescindible de futuro; la mayor o menor discrepancia entre predicciones diversas indican el grado de acuerdo o riesgo que un usuario tiene de aceptar cualquiera de ellas”.

\subsection{Modelos de predicción de series temporales}

La tipología de información disponible, cualitativa o cuantitativa, en el proceso de predicción económica condiciona la selección de las técnicas o modelos más apropiados. En el caso de la información cuantitativa, los datos pueden referirse a un fenómeno o variable aislado en el tiempo, o bien a información estadística referida a diferentes variables entre las que se supone existe algún tipo de relación o conexión en términos de causa y efecto. El primer enfoque es propio del análisis de series temporales, en datos de alta frecuencia, con la finalidad de predecir el futuro basándose en una cierta ley estadística o patrón que puede deducirse del comportamiento históricamente observado (Gujarati, 2004). Las técnicas/modelos de predicción aplicadas a datos de alta frecuencia se utilizan en un horizonte de predicción de corto plazo (proyecciones inferiores a un año). Klein y Kushnirsky (2005) abogan por la utilización de los modelos de alta frecuencia en combinación con sistemas de baja frecuencia para la elaboración de predicciones a más largo plazo: “en esta interacción, los 
modelos de alta frecuencia se emplearían para ajustar los modelos de baja frecuencia a los periodos más recientes $\mathrm{y}$, posteriormente, realizar las estimaciones de largo plazo".

En la Tabla 1 resumimos las principales técnicas/modelos a emplear en el proceso de predicción con series temporales con atención a la diferencia del horizonte de predicción. En todo caso, la combinación de técnicas/modelos, en función de la información disponible, posibilita la obtención de predicciones alternativas. Atendiendo a la recomendación de Pulido (2016): "tener una única predicción en momentos de fuerte cambio puede resultar especialmente peligroso... para predictores y usuarios. No es un problema de cobardía, debilidad de análisis o inseguridad. Es una exigencia de un entorno cambiante y pleno de riesgos que hace posible un variado mosaico de apuestas de futuro”.

Tabla 1

Tipología de técnicas/modelos de predicción con series temporales.

\begin{tabular}{|l|l|}
\hline $\begin{array}{c}\text { Horizonte de } \\
\text { predicción }\end{array}$ & \multicolumn{1}{c|}{ Técnica/Modelo } \\
\hline \multirow{3}{*}{ Corto plazo } & $\begin{array}{l}\text { Medias móviles } \\
\text { Alisado exponencial (Simple, Brown, Holt Winters) } \\
\text { Modelos Autorregresivos integrados de Medias Móviles (ARIMA) } \\
\text { Modelos ARIMA con Análisis de intervención } \\
\text { Función de transferencia, Modelos SARIMAX } \\
\text { Modelos Autorregresivos Condicionales Heteroscedásticos (ARCH, GARCH, EGARCH) }\end{array}$ \\
\hline \multirow{3}{*}{ Medio/largo plazo } & $\begin{array}{l}\text { Ajuste de tendencia } \\
\text { Modelos econométricos uniecuacionales y multiecuacionales } \\
\text { Modelos de Vectores Autorregresivos (VAR) } \\
\text { Modelos de Vectores de Corrección del Error (VEC) }\end{array}$ \\
\hline
\end{tabular}

Fuente: Elaboración propia.

\section{METODOLOGÍA Y DATOS}

De todas las posibilidades recogidas en la Tabla 1 recurrimos a la modelización de series temporales basadas en modelos ARIMA y sus variantes. Una primera aportación de los modelos ARIMA para el pronóstico del tráfico aéreo de pasajeros fue realizado por Box y Jenkins (1976) aplicado a una serie mensual (periodo 1949-1960) de pasajeros internacionales en el aeropuerto de San Francisco, con diferencias de primer orden no estacional y estacional y con componentes de promedios móviles no estacionales y estacionales de orden 1 . Este modelo, denominado de líneas aéreas, se incorpora en algunos programas informáticos como procedimiento automático de estimación pero no significa que siempre sea la mejor elección.

Seguimos la metodología habitualmente empleada para abordar las predicciones del tráfico aéreo mensual de pasajeros a corto plazo, por las características que presentan las series correspondientes. En concordancia con otros trabajos (Tsui et al., 2014; Williams, 2007), planteamos una mejora en la 
modelización consistente en incorporar a la especificación de un modelo ARIMA una variable explicativa externa (función de transferencia), modelo que se conoce como SARIMAX cuando se aplica a series con estacionalidad. Utilizamos los datos de series mensuales de pasajeros comerciales en España procedentes de AENA, cuyas estadísticas de tráfico aéreo se encuentran disponibles a través de su propia página web (www.aena.es), en el periodo comprendido desde enero de 2004 a diciembre de $2015^{1}$ para los 48 aeropuertos que integran la red española. Como elemento de mejora adicional, respecto a otros trabajos similares, examinamos si, y en qué medida, el poder predictivo del tráfico aeroportuario de pasajeros en España puede ser mejorado mediante el uso de información más detallada e individualizada, a través de un enfoque desagregado (suma de las predicciones de los diferentes aeropuertos regionales) frente a la opción recurrente de realizar predicciones agregadas para el conjunto nacional.

Abordamos, en primera instancia, las consideraciones generales respecto al empleo de un enfoque agregado frente a la posibilidad de una aproximación desagregada, para adentrarnos a continuación en la modelización de los datos objeto de análisis.

\subsection{El enfoque agregado vs desagregado en la predicción}

La predicción del tráfico aéreo nacional de pasajeros puede contemplarse como un todo en sí mismo o como agregado de las predicciones realizadas a los datos diferenciados por aeropuertos regionales (cuya suma origina el dato nacional). Se consideran dos enfoques de aproximación a los valores de predicción que dependen del punto de partida adoptado:

1) agregado, de arriba-abajo, top-down, que otorga primacía a la solución agregada directa que significa distribuir el dato nacional por regiones;

2) desagregado, de abajo-arriba, bottom-up, que implica obtener el dato nacional por agregación de los datos regionales.

La aplicación de uno u otro enfoque no proporciona los mismos resultados y la conveniencia de su elección depende del objeto de estudio, uso y expectativas. Existen ventajas y desventajas tanto para el enfoque top-down como para el método de estimación bottom-up. Klein (1969) ya defendía la aproximación topdown que garantiza que las predicciones regionales sean consistentes con las predicciones nacionales: "Para ir de lo general a lo particular es manejable y factible. Hay modelos nacionales viables en existencia... Los modelos nacionales ya han demostrado su eficacia en pruebas repetidas, y es probable que los

\footnotetext{
${ }^{1}$ Las estadísticas oficiales del gestor aeroportuario AENA se inician en el año 2004, y en el desarrollo de la modelización abordada en el presente trabajo el último año completo disponible es el año 2015.
} 
resultados mucho más pobres se obtienen tratando de construir sistemas interregionales completos a partir de una base de datos mucho más débil”. Pero también señalaba la inconveniencia de la no retroalimentación: la vuelta de la región a la nación o entre regiones, que, sin embargo, es la principal ventaja de los modelos desagregados. Pulido (2007) sostiene que "la buena predicción económica sabe mucho de combinar ideas de escuelas muy diferentes de pensamiento; del peso de los condicionantes político-sociales; de la exigencia de combinar enfoques bottom-up y top-down con un enfoque globalizador". No obstante, abundando en las ventajas e inconvenientes de uno u otro planteamiento, López (2015) expone el efecto “caja negra”, que supone un enfoque top-down y las ventajas explicativas de un modelo bottom-up, concluyendo que "hay una tendencia para apoyar los modelos top-down debido a sus menores costes de desarrollo, en términos de coste-eficacia de los resultados".

\subsection{El tráfico aeroportuario de pasajeros en España}

El tráfico aéreo de pasajeros es una de las principales magnitudes de actividad económica de un país. Tiene especial relevancia en la consideración de los flujos económicos con el resto del mundo, máxime en un país como España, destino turístico internacional de primera magnitud, concretamente el tercero, tanto por el número de llegadas de turistas internacionales, como de ingresos por turismo, y primer país del mundo en Travel and Tourism Competitiveness Index (UNWTO, 2016; WEF, 2017).

Más allá de la justificación basada en su aportación directa a la producción interior del país, el transporte aéreo de pasajeros, por su condición de sector intermedio, resulta determinante en el desarrollo de numerosas ramas de actividad, destacando las relacionadas con el turismo: por motivo de ocio y vacaciones, de negocios, compras o cualquier otra razón que motive ${ }^{2}$ un desplazamiento interurbano de personas fuera de su entorno habitual ${ }^{3}$ por un período de tiempo consecutivo inferior a un año.

La estimación de los tráficos futuros de pasajeros en el conjunto de aeropuertos del país (enfoque agregado) constituye, en consecuencia, un desempeño fundamental de predicción económica desde la perspectiva de la evolución coyuntural de la economía. También lo es en clave estructural, con la intención de conocer el grado de consolidación del liderazgo turístico del país.

\footnotetext{
2 Las Notas Metodológicas de la Organización Mundial del Turismo, conforme a las recomendaciones internacionales aprobadas por Naciones Unidas, identifican los siguientes motivos de viaje: vacaciones, recreo y ocio; negocios y motivos profesionales; visitas a familiares y amigos; educación y formación; salud y atención médica; religión/peregrinaciones; compras; tránsito y otros motivos personales (UNWTO, 2013).

${ }^{3}$ Entorno habitual entendido como la "zona geográfica (aunque no necesariamente contigua) en la que una persona realiza sus actividades cotidianas habituales” (UNWTO, 2013).
} 
De hecho, unas predicciones fiables de tráfico aéreo de pasajeros conforman un componente integral para la planificación a corto y a largo plazo y para las decisiones a adoptar en el desarrollo de las infraestructuras aeroportuarias y redes de vuelo (Tsui et al., 2014). Las previsiones a corto plazo para la demanda serán muy útiles para la toma de decisiones en las operaciones inmediatas del aeropuerto, así como las previsiones a largo plazo se pueden utilizar para la planificación estratégica y las inversiones de mayor envergadura (Priyadarshana et al., 2015).

No obstante, resulta igualmente de interés la predicción de los tráficos individuales de pasajeros en los distintos aeropuertos comerciales de la red española (enfoque desagregado), no sólo como medida de control de la precisión alcanzada en la estimación agregada, también porque puede ser un elemento determinante de las diferencias territoriales observadas en el crecimiento económico, a escala de las distintas Comunidades Autónomas y provincias del país. De hecho, en España, los distintos aeropuertos pueden competir entre sí por captar tráficos, como resultado de las estrategias de crecimiento económico regionales o locales.

\section{Figura 1}

Evolución de la cuota de pasajeros aéreos comerciales (principales aeropuertos)

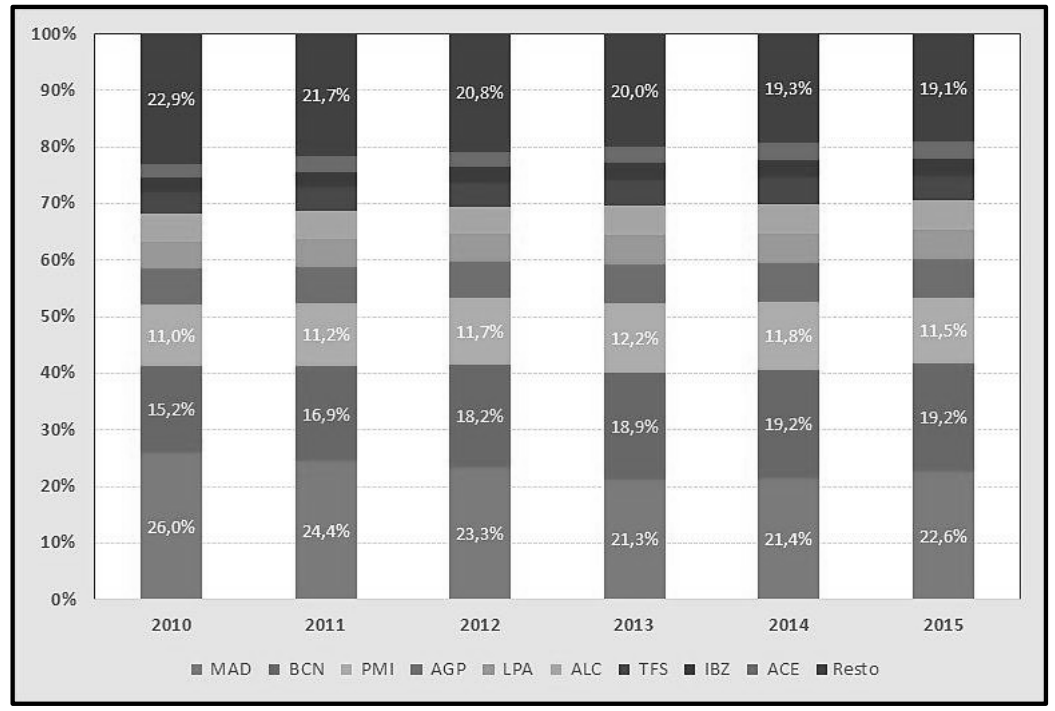

Fuente: Elaboración propia a partir de datos de AENA.

La red de aeropuertos españoles, de gestión centralizada y conjunta, representa un caso un tanto particular a nivel mundial entre los principales mercados aéreos 
internacionales, ya que una misma entidad, $\mathrm{AENA}^{4}$, es la encargada de administrar todas las instalaciones aeroportuarias, adoptando las decisiones relativas a la inversión en la red, las fuentes de financiación, los slots aeroportuarios, etc. (Bel y Fageda, 2011). Entre los aeropuertos que componen la red existen diferencias notables en cuanto a tamaño, capacidad y número de pasajeros transportados. Por una parte, dos hubs importantes, Madrid y Barcelona, en los que se concentra el grueso de las operaciones de varias compañías aéreas, y que sirven como centros distribuidores del tráfico, tanto en el ámbito nacional como internacional. De otro lado, un grupo de aeropuertos eminentemente turísticos, que también cuentan con un peso específico significativo dentro de la red (véase Figura 1). Así, por ejemplo, en el año 2015 alrededor del $80 \%$ del tráfico total de pasajeros comerciales se acumula prácticamente en 9 aeropuertos -Madrid, Barcelona, Palma de Mallorca, Málaga, Alicante, Gran Canaria, Tenerife Sur, Ibiza y Lanzarote-.

La Figura 2 muestra la evolución mensual del tráfico de pasajeros comerciales de toda la red de aeropuertos españoles controlados por AENA, así como las tasas de crecimiento interanual para todo el periodo considerado en este trabajo (2004-2015). Se aprecia el impacto negativo que tuvo la crisis económica sobre la evolución del tráfico aéreo de pasajeros durante los años 2008 y 2009, con descensos que superan en algunos meses el 15\%, así como una nueva caída en los años 2012-2013, con tasas de crecimiento negativas cercanas al 10\%. A partir de octubre de 2013, se vuelve a retomar la senda de crecimiento con incrementos medios en torno al 4\%, y acelerando los ritmos de variación en los dos últimos años de la mano de la recuperación económica y del turismo, tanto externa como internamente.

El comportamiento mensual del tráfico aéreo de pasajeros en la red de aeropuertos españoles presenta una fuerte componente estacional. De manera general, esta pauta se presenta en casi todos los aeropuertos que componen la red, debido a la intensa influencia del tráfico turístico por motivo vacacional en España, especialmente, durante los meses de verano. Este patrón es más acusado y afecta, en mayor medida, a los aeropuertos cuyo tráfico es más dependiente del sector turístico denominado de "sol y playa", como pueden ser los aeropuertos de las Islas Baleares y Canarias (aunque los aeropuertos de Gran Canaria y Tenerife Sur presentan un "pico" de actividad en los meses de invierno), así como los aeropuertos de Málaga y Alicante.

Con el propósito de realizar un análisis ex-post y evaluar el desempeño de los diferentes modelos, en términos de precisión y fiabilidad, se reserva un año de la muestra. De esta forma, las series de datos se dividen en dos submuestras.

\footnotetext{
${ }^{4}$ Desde el 11 de febrero de 2015, AENA cotiza en Bolsa y 49\% de su capital se encuentra en manos privadas, el otro 51\% restante está administrado por el Estado a través de la entidad pública empresarial ENAIRE.
} 
La primera incluye datos que abarcan el periodo comprendido desde enero de 2004 hasta diciembre de 2014, que se utiliza para estimar y ajustar los diferentes modelos. Y la segunda muestra, que abarca desde enero de 2015 hasta diciembre de 2015, cuyos datos se emplean para evaluar el desempeño de los pronósticos arrojados por los distintos modelos estimados.

Figura 2

Pasajeros aéreos comerciales totales red AENA (millones y tasas de variación)

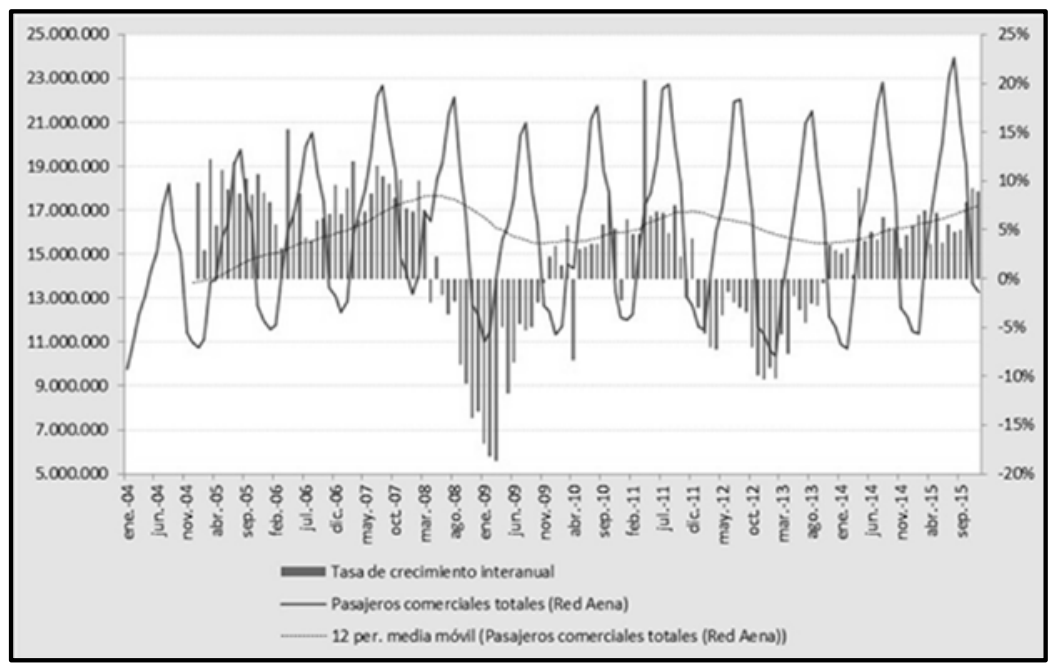

Fuente: Elaboración propia a partir de datos de AENA.

Como hemos comentado previamente, planteamos la utilización de modelos del tipo SARIMAX para la modelización y previsión del tráfico aéreo de pasajeros comerciales, que combinan la modelización univariante ARIMA con una variable explicativa externa, conformando una función de transferencia. La incorporación de una variable explicativa que esté íntimamente relacionada con el tráfico aéreo de pasajeros contribuye a la mejora de la precisión del pronóstico (Williams, 2007; Tsui et al., 2014; Tsui y Balli, 2017).

Los modelos ARIMA, o también denominados de Box y Jenkins por su origen, se construyen empíricamente a partir de la propia serie temporal observada y, básicamente, dependen de tres componentes subyacentes e inherentes al proceso. El término autorregresivo (AR), el integrado (I) y el de media móvil (MA). En la práctica, el procedimiento consiste en encontrar el orden $(p)$ para el proceso autorregresivo, el orden $(q)$ para el proceso de media móvil, y un nivel de diferenciación (d) que, en conjunto, permita construir el modelo que se ajuste mejor a la serie histórica de datos y que, además, pueda realizar predicciones de una manera certera. El proceso integrado $d$ indica el 
grado de diferenciación que se requiere para que la serie sea estacionaria, condición indispensable en los modelos ARIMA. ${ }^{5}$

Los modelos ARIMA estacionales $(P, D, Q)$ complementan el modelo general no estacional ARIMA $(p, d, q)$, desarrollados para captar los patrones estacionales trimestrales o semestrales presentes en las series temporales (Box et al., 2008). La combinación de modelos ARIMA $(p, d, q)$ no estacionales con ARIMA $(P, D, Q)$ estacionales conduce al modelo SARIMA $(p, d, q) \times(P, D, Q)$, también conocido como ARIMA multiplicativo. En forma agregada, su representación general es:

$$
(1-B)^{d}\left(1-B^{s}\right)^{D} Y_{t}=\alpha+\frac{\left(1-\theta_{1} B-\ldots-\theta_{q} B^{q}\right)\left(1-\Theta_{1} B^{s}-\ldots-\Theta_{Q} B^{s Q}\right)}{\left(1-\varnothing_{1} B-\ldots-\varnothing_{p} B^{p}\right)\left(1-\Phi_{1} B^{s}-\ldots-\Phi_{P} B^{s P}\right)} \varepsilon_{t}
$$

donde: $d=$ número de diferencias regulares, $D=$ número de diferencias estacionales, $s=$ amplitud estacional, $\alpha=$ constante óptima, $q=$ número de componentes de medias móviles, $Q=$ número de componentes de medias móviles estacionales, $\theta$ 's=coeficientes de medias móviles, $\Theta$ 's $\mathrm{s}=$ coeficientes de medias móviles estacionales, $p=$ número de componentes autorregresivos, $P=$ número de componentes autorregresivos estacionales, $\emptyset$ 's=coeficientes de los procesos autorregresivos, $\Phi$ 's=coeficientes de los procesos autorregresivos estacionales.

Para considerar el efecto que sobre la serie $Y_{t}$ tendría la inclusión de una o más variables exógenas, en un modelo con componentes estacionales, consideramos los modelos SARIMAX $(p, d, q) \times(P, D, Q)$, que adoptan la forma (asumiendo que la serie $Y_{t}$ es estacionaria):

$$
\varnothing(B) \Phi\left(B^{s}\right) Y_{t}=\alpha+\theta(B) \Theta\left(B^{s}\right) \varepsilon_{t}+X_{t}{ }_{t} \beta
$$

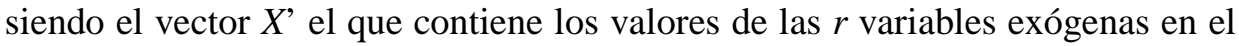
tiempo $t$, cuyos coeficientes están expresados por $\beta$.

En el caso que nos concierne, se propone incluir como variable exógena en el modelo una serie temporal que reproduzca la actividad económica en su conjunto. En concreto, y motivado por su disponibilidad e idoneidad, se empleará el Indicador Sintético de Actividad (ISA), de periodicidad mensual, elaborado por el Ministerio de Economía y Competitividad (Figura 3). Este índice está construido con base en diferentes indicadores parciales, y su ritmo de variación interanual, a partir de datos trimestrales, sigue una evolución muy próxima al PIB, por lo que refleja con bastante exactitud las fases cíclicas de la macromagnitud referida. El transporte aéreo tiene una correlación significativa

\footnotetext{
${ }^{5}$ La estimación de los parámetros de un modelo ARIMA exige que el proceso estocástico subyacente que generó la serie no cambie con respecto al tiempo. Si las características del proceso estocástico varían a lo largo del tiempo, se dice que el proceso no es estacionario. La estacionariedad de una serie implica que la media y la varianza del proceso sean constantes. En este sentido, se requiere que la serie no presente tendencia y que, además, tenga un grado de dispersión similar en cualquier momento del tiempo.
} 
con la actividad económica en su conjunto medida a través del PIB. En consonancia, Tsui et al. (2014) emplean modelos de tipo ARIMAX para predecir el tráfico aéreo de pasajeros en el Aeropuerto Internacional de Honk-Kong, y emplean el PIB per cápita como variable exógena proxy.

Figura 3

Indicador Sintético de Actividad y Tráfico aéreo pasajeros tasas variación interanual

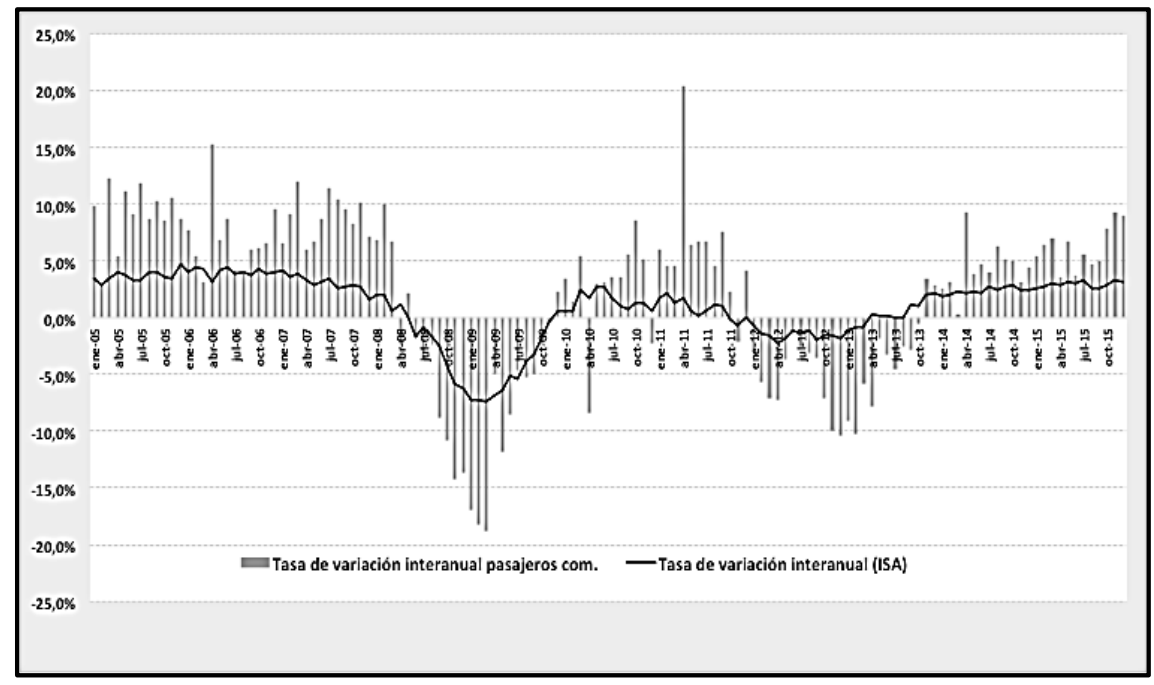

Fuente: Elaboración propia a partir de datos de AENA y del Ministerio de Economía y Competitividad.

\section{MODELOS Y RESULTADOS}

\subsection{Predicción agregada de la serie de pasajeros aéreos comerciales}

Con el propósito de dilucidar cuál de las dos aproximaciones teóricas -enfoque agregado vs. enfoque desagregado- produce estimaciones más precisas, es necesario obtener, en primera instancia, los resultados de ese modelo agregado de pasajeros aéreos comerciales, que posteriormente será comparado con la suma de los pronósticos individuales obtenidos para cada aeropuerto. Por tanto, el primer paso consistirá en obtener una estimación de los pasajeros aéreos comerciales totales a nivel agregado bajo el mismo criterio y metodología que serán empleados en la modelización desagregada por aeropuerto.

Un primer análisis exploratorio de la Figura 2, mostrada previamente, permite comprobar que la varianza de la serie no es constante a lo largo del tiempo, se aprecia una mayor dispersión en los últimos años. También se observa que los pasajeros aéreos comerciales se incrementan con el paso del tiempo, lo que sugiere una tendencia positiva y creciente a lo largo del periodo de estudio 
considerado. Estos elementos podrían ser un indicador preliminar de no estacionariedad de la serie, tanto en media como en varianza. Con el correspondiente diagrama de rango-media para un subconjunto de observaciones se puede confirmar la no estacionariedad en varianza de la serie de pasajeros aéreos comerciales totales en España.

Figura 4

Diagrama Rango-Media de los pasajeros aéreos comerciales totales

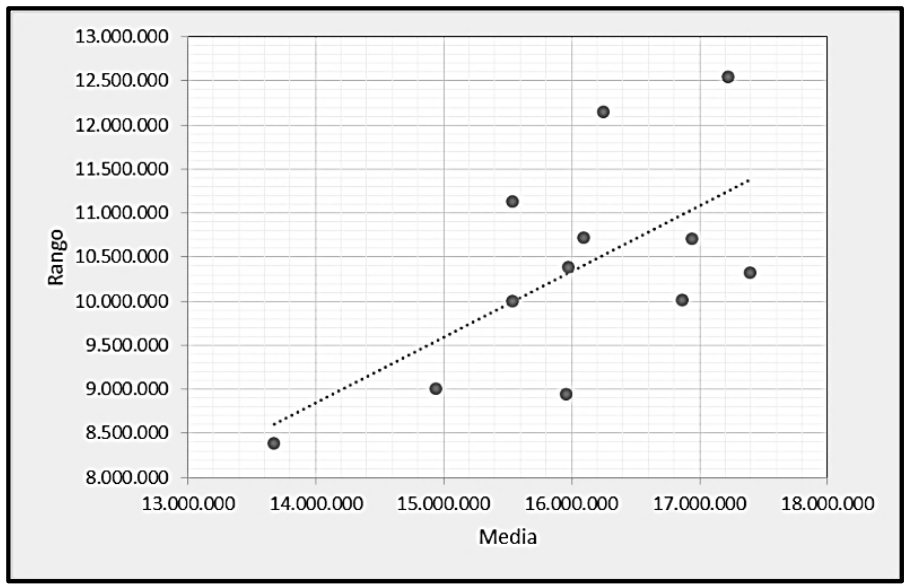

Nota: utilizando 12 submuestras de tamaño 12, pendiente de 'rango' con respecto a 'media' $=0.747968$, el valor $p$ para $H_{0}$ : pendiente $=0$ es 0.0271281

Fuente: Elaboración propia a partir de datos de AENA

Tabla 2

Prueba de raíz unitaria de Dickey-Fuller Aumentada (ADF)

(Diferencia del logaritmo de los pasajeros aéreos comerciales totales, periodo 2004-2014)

\begin{tabular}{|c|c|}
\hline Prueba & Estadístico $t$ \\
\hline Dickey-Fuller Aumentada (ADF) & $-1.929961^{\star}$ \\
\hline
\end{tabular}

Nota: * al 5\% de significatividad estadística. Variable exógena: ninguna. Número de retardos $=12$ selección automática basada en SIC.

Fuente: Elaboración propia.

Para disminuir la variabilidad de la serie, así como para eliminar la tendencia de la misma, aplicamos una diferencia regular al logaritmo neperiano de los datos. Las correspondientes funciones de autocorrelación (FAC) y autocorrelación parcial (FACP) de dicha transformación posibilitan la observación de los coeficientes que, en el caso de la FAC, muestran un patrón oscilante, indicativo de no estacionariedad, mientras que en la FACP existen coeficientes significativos en los retardos estacionales (12). El procedimiento más utilizado para contrastar la estacionariedad de una serie es la prueba de Dickey-Fuller (1979). Siguiendo la pauta recogida en Tsui et al. (2014) y Nasiru et al. (2013), 
recogemos en la Tabla 2 los resultados principales de la prueba Dickey-Fuller Aumentada (ADF) sobre esta primera transformación, donde se confirma que la serie aún no presenta un patrón estacionario.

Como se ha mencionado (Figura 2), la estacionalidad (ciclos de determinada duración que se completan al menos una vez en el año) está claramente presente en la serie, con puntas en los meses de verano y valles en los meses de enero y febrero. La Figura 5 de las subseries estacionales muestra esta situación en las secciones similares de las estaciones: picos en los meses de julio y agosto, indicando periodos de alta intensidad de viajes durante las vacaciones de verano, seguidos por una disminución progresiva hasta alcanzar mínimos en los meses de invierno.

Figura 5

Subseries estacionales de los pasajeros aéreos comerciales totales (2004-2014)

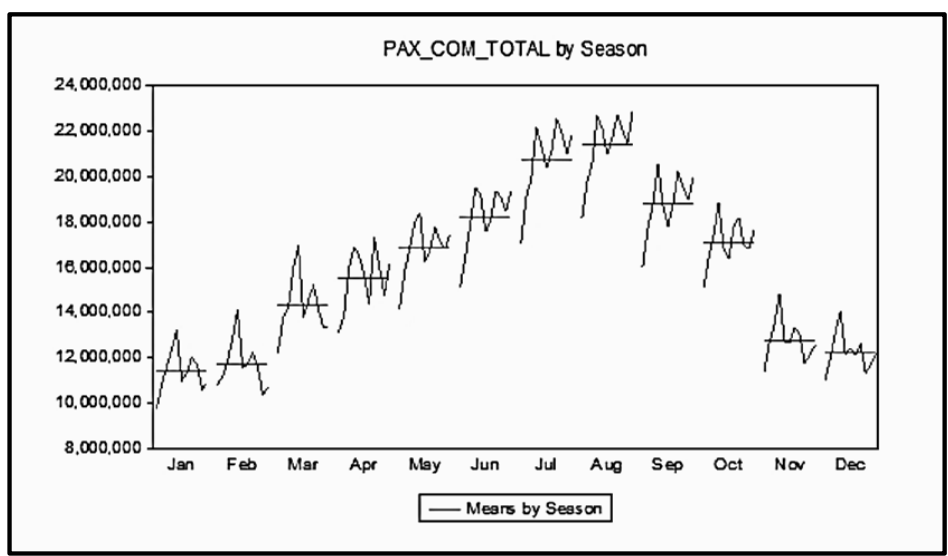

Fuente: Elaboración propia.

Del análisis exploratorio deducimos que es preciso realizar una transformación de la serie de pasajeros aéreos comerciales en España, para delinear un comportamiento estable en media y varianza. Cuando una serie presenta una raíz unitaria en la frecuencia cero se convierte en estacionaria aplicando una primera diferencia. Las raíces unitarias estacionales se corrigen aplicando una diferenciación estacional, filtrando la serie con el operador de diferencia estacional $\left(1-B^{s}\right)$.

Como tenemos evidencia gráfica de la posible presencia de raíces unitarias estacionales recurrimos a la prueba HEGY desarrollada por Hylleberg et al. (1990), extendida por Beaulieu y Miron (1992) para datos mensuales, que es una generalización de la prueba aumentada de Dickey-Fuller y que permite contrastar la estacionariedad en la frecuencia cero (regular) y en las frecuencias estacionales al mismo tiempo (Tabla 3). Permite decidir cuáles de las frecuencias 
estacionales corresponden a raíces estacionales significativas. Junto a las pruebas t y F para las distintas frecuencias, el test incluye una prueba $\mathrm{F}$ de significación conjunta para todas las frecuencias estacionales y otra para todas las frecuencias incluyendo la frecuencia cero. Los resultados del test HEGY permiten concluir que no se puede rechazar la hipótesis de raíz unitaria en la frecuencia cero y la presencia de raíces unitarias estacionales en la frecuencia estacional.

Tabla 3

Resultados del test HEGY (raíces unitarias presentes en datos mensuales) (serie logaritmo de los pasajeros aéreos comerciales totales periodo 2004-2014)

\begin{tabular}{|c|c|c|c|c|}
\hline Notación y Frecuencia & Nombre de la raíz & $\begin{array}{c}\text { Número de } \\
\text { ciclos por año }\end{array}$ & Estadístico & p-valor \\
\hline$\pi 1: 0$ & no estacional & 0 & $\mathrm{t}_{1}=-3,64$ & 0,02060 \\
\hline$\pi 2: \pi$ & bimensual & 6 & $\mathrm{t}_{2}=-4,76$ & 0,00097 \\
\hline$\pi 3 \pi 4: \pi / 2$ & cuatrimestral & 3 & $\mathrm{~F}_{3}=5,67$ & 0,17700 \\
\hline$\pi 5 \pi 6: 5 \pi / 6$ & -- & 5 & $\mathrm{~F}_{5}=20,94$ & 0,00000 \\
\hline$\pi 7 \pi 8: 2 \pi / 3$ & trimestral & 4 & $\mathrm{~F}_{4}=4,09$ & 0,37525 \\
\hline$\pi 9 \pi 10: \pi / 6$ & anual & 1 & $\mathrm{~F}_{1}=0,82$ & 0,96128 \\
\hline$\pi 11 \pi 12: \pi / 3$ & semestral & 2 & $\mathrm{~F}_{2}=9,87$ & 0,01739 \\
\hline$\pi 2 a \pi 12$ & todas estacionales & & $\mathrm{F}_{\mathrm{s}}=10,00$ & -- \\
\hline$\pi 1 a \pi 12$ & todas estacionales $\mathrm{y}$ & & $\mathrm{F}_{\mathrm{t}}=11,12$ & -- \\
\hline
\end{tabular}

Fuente: Elaboración propia.

Procedemos a la transformación de una diferencia en la parte regular y una diferencia en la parte estacional ( $\mathrm{s}=12$ ) de la serie en logaritmos neperianos de pasajeros aéreos comerciales y realizamos la prueba ADF a la serie transformada para comparar con los resultados previos, verificando la estacionariedad (Tabla 4).

Tabla 4

Prueba de raíz unitaria de Dickey-Fuller Aumentada (ADF)

(Diferencia regular y estacional del logaritmo de los pasajeros aéreos comerciales totales, periodo 2004-2014)

\begin{tabular}{|c|c|}
\hline Prueba & Estadístico $t$ \\
\hline Dickey-Fuller Aumentada (ADF): & $-17.83033^{*}$ \\
\hline Nota: $\begin{array}{c}* \text { al } 5 \% \text { de significatividad estadística. Variable exógena: ninguna. Número de } \\
\text { retardos }=0, \text { selección automática basada en SIC. }\end{array}$
\end{tabular}

Fuente: Elaboración propia.

La serie transformada presenta las condiciones necesarias para identificar y estimar el mejor modelo que recoja su comportamiento histórico. En este punto, varios trabajos sobre la demanda de transporte aéreo incorporan el análisis de intervención y tratan de dilucidar el impacto que ciertos acontecimientos de carácter externo han tenido sobre las series de pasajeros. Por ejemplo, Min, Kung 
y Liu (2011) para el caso de Taiwán, o para el ámbito español las investigaciones realizadas por Coto-Millán et al. (2004) e Inglada y Rey (2004). A partir de aquí, es posible detectar ciertos eventos extraordinarios que han impactado al sector del transporte aéreo en España durante el periodo de estudio considerado. En el periodo analizado, destacamos la huelga de controladores de diciembre de 2010 y la erupción del volcán islandés Eyjafjallajökull que obligó a cerrar el espacio aéreo europeo en abril de 2010, acontecimientos que afectaron negativamente a la serie de pasajeros aéreos comerciales. Por tanto, la modelización incluye, además, estas dos variables de evento, que en el caso de la huelga de controladores (DHCO) toma el valor de 1 en el mes de diciembre de 2010 y cero en los demás casos, y para la erupción del volcán islandés (DVOL) la variable considera un valor de 1 en el mes de abril de 2010 y cero en los otros meses, tal que:

$$
\text { DHCO }=\left\{\begin{array}{l}
1, t=2010(12) \\
0, \text { cualquier otro caso }
\end{array} ; D V O L=\left\{\begin{array}{l}
1, t=2010(4) \\
0, \text { cualquier otro caso }
\end{array}\right.\right.
$$

Después de estudiar la estructura de los correlogramas y las correspondientes funciones de autocorrelación (ACF) y autocorrelación parcial (PACF) sobre la serie transformada, así como la estructura de los residuos, se encuentra el modelo SARIMAX que mejor se ajusta y predice la serie de pasajeros aéreos comerciales totales en su variante agregada (Tabla 5).

Tabla 5

Coeficientes y parámetros del modelo SARIMAX (ISA) de los pasajeros comerciales, enfoque agregado

\begin{tabular}{|c|c|}
\hline Variable dependiente: & D(LOG(PAX_COM_TOTAL)) \\
\hline \hline Variable & \\
\hline \hline D(LOG(ISA)) & $\begin{array}{c}1.816799 \\
(0.330485)\end{array}$ \\
\hline D(DHCO) & -0.049467 \\
$(0.020772)$ \\
\hline D(DVOL) & -0.101078 \\
& $(0.020748$ \\
\hline MA(1) & -0.533171 \\
& $(0.083467$ \\
\hline SMA(12) & -0.342577 \\
& $(0.093257)$ \\
\hline $\mathrm{R}^{2}$ & 0.600154 \\
\hline ser & 0.028734 \\
\hline AIC & -4.220349 \\
\hline SIC & -4.103579 \\
\hline Durbin-Watson & 2.007352 \\
\hline
\end{tabular}

Nota: AlC: Criterio de información de Akaike,

SIC: Criterio de información de Schwarz.

Fuente: Elaboración propia. 


\subsection{Predicción desagregada de la serie de pasajeros aéreos comerciales}

Es preciso señalar que el tráfico aéreo en España está bastante concentrado en pocos aeropuertos y existe un grupo de aeropuertos cuyos niveles de tráfico son relativamente bajos en relación al total, y en algunos casos, además, los datos presentan incluso cierta volatilidad al ser analizados. Por tanto, la aplicación directa de la metodología descrita puede resultar, en ciertas ocasiones, inviable o con resultados poco satisfactorios. A este respecto, Airports Council International, ACI (2011) establece que aunque sea difícil de cuantificar con precisión el tamaño que debería tener un aeropuerto para beneficiarse de un enfoque econométrico de predicción, serían, generalmente, más apropiados aeropuertos con niveles de tráfico iguales o superiores al millón de pasajeros por año. La razón principal es que los aeropuertos más pequeños, con menor tráfico, son, a menudo, más sensibles a las estrategias de una única línea aérea o al arbitrio de un número limitado de segmentos de tráfico o mercados. Aeropuertos más grandes y más diversificados son más idóneos para la aplicación de la técnica econométrica.

Por ello, siguiendo esta recomendación, se ha optado por separar de la muestra desagregada los aeropuertos cuyo nivel de tráfico promedio en los últimos seis años (2010-2015) haya sido inferior al millón de pasajeros. Los datos correspondientes se agregan y se consideran como un único aeropuerto, a efectos de su posterior modelización. Con esta operación, se consideran 20 aeropuertos con más de un millón de pasajeros promedio en los últimos seis años y que aglutinan, igualmente, en promedio el $95,9 \%$ del total de los pasajeros comerciales de la red en dicho periodo (Tabla 6). Por tanto, los 28 aeropuertos restantes con niveles de tráfico promedio inferiores al millón de pasajeros en los últimos años, y que se considerarán de ahora en adelante como un único aeropuerto, representan tan sólo el 4,1\% restante.

A partir de esta segmentación se procede a la estimación individual de los aeropuertos bajo las mismas condiciones para su posterior agregación ${ }^{6}$. Se considera el mejor modelo ARIMA, SARIMA encontrado, se emplean las mismas variables exógenas y de evento, y se evalúa individualmente su inclusión o descarte, de acuerdo a su significatividad estadística. Las estimaciones

\footnotetext{
${ }^{6}$ Se han estimado individualmente los 20 aeropuertos con un tráfico promedio en los últimos años mayor o igual al millón de pasajeros, más un aeropuerto denominando "resto" que engloba a todos los demás aeropuertos, con un volumen de tráfico menor a un millón de pasajeros, y que a efectos prácticos se considera como un solo aeropuerto. El grupo de aeropuertos "resto" incluye aeropuertos que se aproximan al millón de pasajeros junto con otras instalaciones aeroportuarias cuyos niveles de tráfico son muy pequeños y excesivamente volátiles. Al tratarlos como una sola entidad, la modelización se vuelve viable, siguiendo los criterios de ACI. La suma de los 29 aeropuertos restantes alcanza en promedio casi 8 millones en pasajeros en el periodo 20102015. Además, dado que el objetivo principal del trabajo es predecir el tráfico total de pasajeros aéreos a nivel nacional, es imprescindible la consideración de todos los aeropuertos en el análisis.
} 
individuales para cada aeropuerto reflejan que la variable explicativa utilizada (ISA) es significativa, al menos al $10 \%$ de significatividad estadística, en todos los casos excepto en tres aeropuertos (Fuerteventura, Murcia y Reus). Las variables dummy de evento (DHCO y DVOL) no resultan estadísticamente significativas en todos los aeropuertos. Existen algunas estimaciones en las que sólo se incluye alguna de las dos variables o ninguna. ${ }^{7}$

\section{Tabla 6}

Aeropuertos con un nivel de tráfico aéreo mayor y menor a un millón de pasajeros comerciales (promedio 2010-2015)

\begin{tabular}{|c|c|c|c|}
\hline \multicolumn{2}{|c|}{$\begin{array}{c}\text { Aeropuertos tráfico pasajeros } \geq 1 \text { millón } \\
\text { personas }\end{array}$} & \multicolumn{2}{|c|}{ Aeropuertos tráfico pasajeros $<1$ millón personas } \\
\hline $\begin{array}{c}\text { Lanzarote (ACE) } \\
\text { Málaga (AGP) } \\
\text { Alicante (ALC) } \\
\text { Barcelona (BCN) } \\
\text { Bilbao (BIO) } \\
\text { Fuerteventura (FUE) } \\
\text { Girona (GRO) } \\
\text { Ibiza (IBZ) } \\
\text { Gran Canaria (LPA) } \\
\text { Madrid (MAD) } \\
\text { Menorca (MAH) }\end{array}$ & $\begin{array}{c}\text { Murcia (MJV) } \\
\text { Asturias (OVD) } \\
\text { Palma de Mallorca (PMI) } \\
\text { Reus (REU) } \\
\text { Santiago (SCQ) } \\
\text { Sevilla (SVQ) } \\
\text { Tenerife Norte (TFN) } \\
\text { Tenerife Sur (TFS) } \\
\text { Valencia (VLC) }\end{array}$ & $\begin{array}{c}\text { A Coruña (LCG) } \\
\text { Albacete (ABC) } \\
\text { Algeciras (AEI) } \\
\text { Almería (LEI) } \\
\text { Badajoz (BJZ) } \\
\text { Burgos (RGS) } \\
\text { Ceuta (JCU) } \\
\text { Córdoba (ODB) } \\
\text { El Hierro (VDE) } \\
\text { Granada (GRX) } \\
\text { Huesca (HSK) } \\
\text { Jerez (XRY) } \\
\text { La Gomera (QGZ) } \\
\text { La Palma (SPC) } \\
\text { León (LEN) } \\
\text { La Rioja (RJL) }\end{array}$ & $\begin{array}{c}\text { Madrid Cuatro Vientos } \\
\text { (MCV) } \\
\text { Madrid Torrejón (TOJ)* } \\
\text { Melilla (MLN) } \\
\text { Pamplona (PNA) } \\
\text { Sabadell (QSA) } \\
\text { Salamanca (SLM) } \\
\text { San Sebastián (EAS) } \\
\text { Santander (SDR) } \\
\text { Son Bonet (SBO) } \\
\text { Valladolid (VLL) } \\
\text { Vigo (VGO) } \\
\text { Vitoria (VIT) } \\
\text { Zaragoza (ZAZ) }\end{array}$ \\
\hline \multicolumn{2}{|c|}{$\begin{array}{l}20 \text { aeropuertos: representan } 95,9 \% \text { del tráfico total } \\
\text { de pasajeros aéreos comerciales }\end{array}$} & \multicolumn{2}{|c|}{$\begin{array}{l}29 \text { aeropuertos: representan el } 4,1 \% \text { del tráfico total de } \\
\text { pasajeros aéreos comerciales }\end{array}$} \\
\hline
\end{tabular}

Nota: * El Aeropuerto de Torrejón (TOJ) dejó de recibir vuelos civiles a partir del año 2013.

Fuente: Elaboración propia.

En total se estiman 21 modelos, uno para cada aeropuerto modelizable (20) atendiendo al criterio de ACI (2011) y otro referido al aeropuerto "resto" resultante de la suma de los que tienen un tráfico menor (Tabla 6). Una vez obtenidas las estimaciones individuales por aeropuerto (enfoque desagregado) según los modelos aplicados (Tabla 7), se adicionan los resultados y se procede a la comparación con la estimación realizada en la versión agregada.

\footnotetext{
${ }^{7}$ Además, existen otros casos particulares, los aeropuertos de Girona (GRO), Reus (REU) y Valencia (VLC), donde se detectaron varios puntos atípicos que se modelizaron con la inclusión de variables dicotómicas en distintos puntos de la serie.
} 
Tabla 7

Modelos estimados para cada aeropuerto individual

\begin{tabular}{|c|c|}
\hline $\begin{array}{l}\text { Series individuales de aeropuertos } \\
\text { (pasajeros comerciales) }\end{array}$ & Modelos estimados \\
\hline D(LOG(PAX_COM_ACE)) & $(0,1,1)(0,1,1)_{12}$ \\
\hline D(LOG(PAX_COM_AGP)) & $(0,1,4)(0,1,1)_{12}$ \\
\hline D(LOG(PAX_COM_ALC)) & $(0,1,1)(0,1,1)_{12}$ \\
\hline D(LOG(PAX_COM_BCN)) & $(0,1,0)(0,1,1)_{12}$ \\
\hline D(LOG(PAX_COM_BIO)) & $(0,1,1)(0,1,1)_{12}$ \\
\hline D(LOG(PAX_COM_FUE)) & $(0,1,1)(0,1,1)_{12}$ \\
\hline D(LOG(PAX_COM_GRO)) & $(0,1,1)(0,1,0)_{12}$ \\
\hline D(LOG(PAX_COM_IBZ)) & $(1,1,1)(0,1,0)_{12}$ \\
\hline D(LOG(PAX_COM_LPA)) & $(0,1,1)(0,1,1)_{12}$ \\
\hline D(LOG(PAX_COM_MAD)) & $(0,1,1)(0,1,1)_{12}$ \\
\hline D(LOG(PAX_COM_MAH)) & $(0,1,2)(0,1,0)_{12}$ \\
\hline D(LOG(PAX_COM_MJV)) & $(0,1,4)(1,1,0)_{12}$ \\
\hline D(LOG(PAX_COM_OVD)) & $(0,1,0)(0,1,1)_{12}$ \\
\hline D(LOG(PAX_COM_PMI)) & $(0,1,4)(0,1,0)_{12}$ \\
\hline D(LOG(PAX_COM_REU)) & $(0,1,4)(0,1,1)_{12}$ \\
\hline D(LOG(PAX_COM_SCQ)) & $(1,1,1)(0,1,1)_{12}$ \\
\hline D(LOG(PAX_COM_SVQ)) & $(0,1,1)(0,1,1)_{12}$ \\
\hline D(LOG(PAX_COM_TFN)) & $(0,1,1)(0,1,1)_{12}$ \\
\hline D(LOG(PAX_COM_TFS)) & $(0,1,1)(0,1,1)_{12}$ \\
\hline D(LOG(PAX_COM_VLC)) & $(0,1,0)(0,1,1)_{12}$ \\
\hline D(LOG(PAX_COM_RESTO)) & $(0,1,0)(0,1,1)_{12}$ \\
\hline D(LOG(PAX_COM_TOTAL)) & $(0,1,1)(0,1,1)_{12}$ \\
\hline
\end{tabular}

Fuente: Elaboración propia.

\subsection{Comparación de resultados}

La predicción económica requiere considerar la fiabilidad o grado de precisión del pronóstico realizado. Fiabilidad que viene condicionada por la metodología utilizada en el proceso de predicción y por el tipo de información disponible, cuantificada recurriendo a criterios económicos y estadísticos. Desde el punto de vista de los criterios económicos, la fiabilidad vendrá determinada por la magnitud concreta obtenida en los datos predichos y su interpretación. Los criterios estadísticos se basan en calcular las medidas de aproximación al dato real, conocido, y valoran el resultado obtenido en términos del error cometido.

En este caso, se trata de comparar la predicción agregada con la procedente de la suma de las predicciones de los aeropuertos individuales, para determinar cuál de los dos enfoques, agregado y desagregado, resulta más preciso y, por tanto, aporta más fiabilidad. Según los criterios estadísticos, atendemos a dos medidas de desempeño: la raíz del error cuadrático medio (RECM), y el porcentaje de error absoluto medio (PEAM) o error porcentual absoluto medio. Este último tiene la ventaja de ser interpretado de manera directa y sencilla 
debido a que está expresado como porcentaje de los valores realizados. Ambos valores están basados en los términos de error de pronóstico $\boldsymbol{e}_{\boldsymbol{t}}$ y se encuentran expresados bajo las siguientes formulaciones:

$$
R E C M=\sqrt{\frac{1}{n} \sum_{t=1}^{n} e_{t}^{2}} \quad \text { PEAM }=\frac{100}{n} \sum_{t=1}^{n} \frac{\left|e_{t}\right|}{y_{t}}
$$

donde: $e_{t}=\left(y_{t}-\widehat{y_{t}}\right)$

Estos criterios son los habituales en cualquier análisis de valoración de la fiabilidad de la predicción y han sido también utilizados en trabajos similares relacionados con series de viajeros internacionales (por ejemplo, Lim y McAleer, 2002). Adicionalmente, para completar la valoración, consideraremos dos criterios más, el error absoluto medio (EAM), y el coeficiente de desigualdad U de Theil (1966).

Tabla 8

Evaluación de los pronósticos de pasajeros aéreos, enfoque agregado vs. enfoque desagregado

\begin{tabular}{|c|c|c|c|}
\hline & $\begin{array}{c}\text { Pasajeros aéreos } \\
\text { Valor } \\
\text { histórico real (miles) } \\
\end{array}$ & $\begin{array}{l}\text { Modelo } 1 \text { agregado } \\
\text { SARIMAX (ISA) }\end{array}$ & $\begin{array}{c}\text { Modelo } 2 \text { desagregado } \\
\text { Suma de aeropuertos } \\
\text { (modelos individuales) }\end{array}$ \\
\hline ene-15 & 11.468 & 11.359 & 11.379 \\
\hline feb-15 & 11.387 & 11.219 & 11.446 \\
\hline mar-15 & 14.299 & 14.191 & 14.336 \\
\hline abr-15 & 16.751 & 16.663 & 16.639 \\
\hline may-15 & 18.655 & 18.265 & 18.199 \\
\hline jun-15 & 20.091 & 20.141 & 20.015 \\
\hline jul-15 & 23.039 & 23.024 & 22.890 \\
\hline ago-15 & 23.932 & 23.551 & 23.527 \\
\hline sep-15 & 20.952 & 20.702 & 20.589 \\
\hline oct-15 & 19.069 & 18.426 & 18.342 \\
\hline nov-15 & 13.694 & 13.203 & 13.291 \\
\hline dic-15 & 13.278 & 12.770 & 12.806 \\
\hline 2015 & 206.615 & 203.515 & 203.458 \\
\hline \multirow{12}{*}{$\begin{array}{l}\text { Medidas } \\
\text { evaluación } \\
\text { error } \\
\text { predicción }\end{array}$} & RECM 3 m. & 131,1 & 65,5 \\
\hline & RECM 6 m. & 188,7 & 199,9 \\
\hline & RECM $12 \mathrm{~m}$. & 333,4 & 350,1 \\
\hline & EAM $3 \mathrm{~m}$. & 128,0 & 62,0 \\
\hline & EAM $6 \mathrm{~m}$. & 152,1 & 138,6 \\
\hline & EAM $12 \mathrm{~m}$. & 266,7 & 279,2 \\
\hline & PEAM 3 m. & $1,06 \%$ & $0,52 \%$ \\
\hline & PEAM 6 m. & $1,01 \%$ & $0,84 \%$ \\
\hline & PEAM $12 \mathrm{~m}$. & $1,64 \%$ & $1,62 \%$ \\
\hline & $\mathrm{U}$ Theil $3 \mathrm{~m}$. & 0,005 & 0,003 \\
\hline & U Theil $6 \mathrm{~m}$. & 0,006 & 0,006 \\
\hline & $\mathrm{U}$ Theil $12 \mathrm{~m}$. & 0,009 & 0,010 \\
\hline
\end{tabular}

Nota: Datos de pasajeros en miles de personas, $3 \mathrm{~m}$ : pronóstico a 3 meses vista, $6 \mathrm{~m}$ : pronóstico a 6 meses vista, $12 \mathrm{~m}$ : pronóstico a 12 meses vista.

Fuente: Elaboración propia. 
En la Tabla 8 se recogen los resultados de la aplicación de los dos modelos planteados para el tráfico aéreo de pasajeros en España: modelo agregado (serie nacional total) y modelo desagregado (series de aeropuertos individuales). Con la finalidad de valorar la precisión de los resultados obtenidos (pronósticos) en términos del error cometido, se contempla una diferenciación en tres periodos (3 meses, 6 meses y a 12 meses vista), aunque este tipo de modelos "funciona" (son más fiables) en horizontes de predicción inferiores a 3 meses. Así se constata en Tsui et al. (2014), donde refieren que los errores de pronóstico aumentan notablemente cuando el horizonte de predicción llega a tres meses. Esto supone que los modelos pueden experimentar errores mayores para pronósticos a largo plazo. Se aprecia que el modelo 2 (modelo desagregado, con predicciones calculadas como suma de aeropuertos individuales) es el que presenta el menor error de predicción calculado con las medidas RECM, EAM y PEAM en el pronóstico a tres meses vista $(65,5,62,0$ y $0,52 \%$, respectivamente frente a valores de 131,1, 128,0 y 1,06\% del modelo agregado). A 6 meses vista, el modelo desagregado es mejor que el modelo agregado en términos de EAM y de PEAM, pero peor según RECM. A 12 meses vista, periodo demasiado amplio para su consideración real (porque se haría una revisión de predicciones antes) el esfuerzo realizado en la desagregación sólo compensaría en términos del PEAM, obteniéndose valores satisfactorios (cercanos a cero) y similares en ambos modelos para el coeficiente de desigualdad U de Theil. De manera global, el modelo en su versión desagregada es ligeramente superior bajo el criterio de desempeño seleccionado del PEAM.

Figura 6

Comparativa valor histórico real vs. pronóstico del modelo 1 (agregado) y modelo 2

(desagregado) Pasajeros aéreos, año 2015

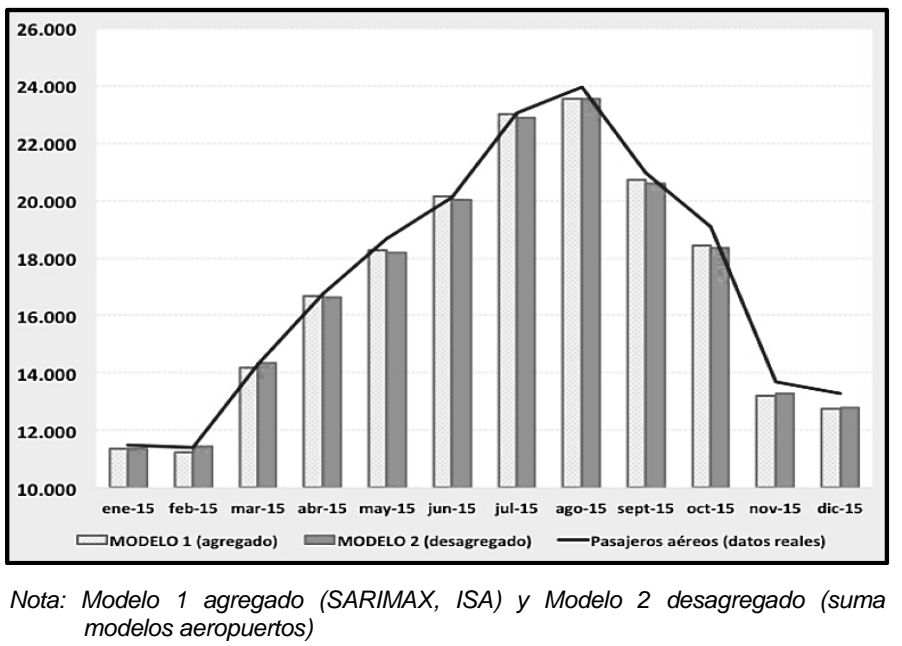

Fuente: Elaboración propia. 
La Figura 6 muestra los valores históricos reales de los datos de pasajeros aéreos frente a los valores pronosticados del enfoque agregado, SARIMAX con ISA (modelo 1), y de la aproximación desagregada a partir de la suma de aeropuertos con los modelos propios (modelo 2) para el detalle mensual del año 2015. En ambos casos, los modelos logran capturar de una manera adecuada la serie en niveles y su respectiva estacionalidad que es el objetivo perseguido.

En términos generales, la previsión de los dos enfoques, agregado, top-down, y desagregado, bottom-up, se ajusta razonablemente bien a los valores históricos (Tabla 9). Ambas estimaciones se encuentran, en promedio, ligeramente por debajo de los valores reales, es decir, infravaloran. Aunque el modelo más alejado tan sólo se queda al 98,5\% del dato real para el promedio del año analizado. En especial, existe una discrepancia mayor en el segundo semestre, donde los errores cometidos por ambos modelos son mayores en dicho periodo. Como ya se puso de manifiesto al analizar los errores, el porcentaje de precisión o grado de concordancia o adecuación (entendido como la cercanía del dato de predicción al dato real) en el promedio de los tres primeros meses de predicción (enero-marzo) es muy elevado, del 100\% en el modelo desagregado.

\section{Tabla 9}

Evaluación de los pronósticos de pasajeros aéreos, enfoque agregado vs. enfoque desagregado, en términos de adecuación de las predicciones por subperiodos

\begin{tabular}{|c|c|c|c|c|c|}
\hline & $\begin{array}{c}\text { Pasajeros } \\
\text { aéreos reales }\end{array}$ & $\begin{array}{c}\text { Predicción } \\
\text { Modelo 1 } \\
\text { agregado }\end{array}$ & $\begin{array}{c}\text { Predicción } \\
\text { Modelo 2 } \\
\text { desagregado }\end{array}$ & $\begin{array}{c}\text { \% Precisión } \\
\text { Modelo 1 }\end{array}$ & $\begin{array}{c}\text { \% Precisión } \\
\text { Modelo 2 }\end{array}$ \\
\hline 2015 & 206.615 & 203.514 & 203.459 & $98,5 \%$ & $98,5 \%$ \\
\hline enero-marzo & 12.385 & 12.256 & 12.387 & $99,0 \%$ & $100,0 \%$ \\
\hline enero-junio & 15.442 & 15.306 & 15.336 & $99,1 \%$ & $99,3 \%$ \\
\hline julio-diciembre & 18.994 & 18.613 & 18.574 & $98,0 \%$ & $97,8 \%$ \\
\hline
\end{tabular}

Fuente: Elaboración propia.

En la mayoría de los trabajos sobre series temporales la práctica habitual es emplear un solo modelo de predicción para pronosticar múltiples horizontes. Sin embargo, hay evidencias que sugieren que el uso de diferentes modelos de predicción para diferentes horizontes podría mejorar la precisión del pronóstico (Kang, 2003).

\section{CONCLUSIONES}

En este trabajo hemos abordado la realización de predicciones económicas desde la perspectiva de la evolución coyuntural de la economía mediante la estimación de los tráficos futuros de pasajeros aéreos en el conjunto de aeropuertos del país (enfoque agregado, top-down), que se ha complementado con la visión que ofrece la predicción de los tráficos individuales de pasajeros en los distintos aeropuertos comerciales de la red española (enfoque desagregado, 
bottom-up) para llegar al dato nacional. Esta diferenciación tiene un claro componente económico, no abordado en nuestro análisis, pero que puede resultar de interés en el sentido de profundizar en las diferencias territoriales observadas.

Por tanto, se puede concluir que bajo estas condiciones y durante el periodo de tiempo seleccionado (año 2015), el enfoque desagregado (bottom-up) construido a partir de la suma de los modelos estimados por aeropuertos individuales, supera en los tres horizontes de predicción establecidos (3 meses, 6 meses y 12 meses) a su "rival" (Hendry y Richard, 1983), agregado (top-down), y bajo el criterio de desempeño del PEAM. Se constata la mejor adecuación del modelo desagregado a tres meses vista. Estos resultados se alinean con las conclusiones similares del trabajo de Carson et al. (2011), para predicciones mensuales del tráfico aéreo de pasajeros en Estados Unidos en el periodo 19902002, donde concluyen que las predicciones son más precisas a partir de un modelo desagregado que a nivel agregado para diferentes periodos de tiempo, basándose en las medidas sobre el error RECM y PEAM. Sostienen que el desempeño de un enfoque desagregado depende del equilibrio entre el grado de heterogeneidad permitido y la incertidumbre de la estimación.

No obstante, hasta el momento, poco se sabe sobre el comportamiento de la predicción individual de los aeropuertos, donde habría que profundizar más incluso en la "calidad" de la información de partida. Podría ser que el resultado esté enmascarando, en cierta forma, subestimaciones o sobrestimaciones cometidas en las estimaciones individuales. El siguiente paso, para abordar en una fase posterior de la investigación, sería comprobar igualmente de una manera formal, que además los pronósticos individuales generados también se comporten mejor que el reparto entre aeropuertos de la predicción agregada, obtenidos, por ejemplo, mediante un modelo de cuotas estimadas.

Finalmente, es importante considerar que la elaboración y difusión de predicciones económicas puede repercutir en el funcionamiento de los mercados financieros, generando expectativas, cuestionar o apoyar las políticas gubernamentales o incluso influir en las decisiones o comportamiento de los agentes económicos. Atendiendo a la recomendación de Pulido (2016), la obtención de predicciones alternativas posibilita una diversidad de apuestas de futuro y, además, abre el camino hacia la combinación de predicciones.

\section{REFERENCIAS BIBLIOGRÁFICAS}

ACI (2011). Airport Traffic Forecasting Manual: A practical guide addressing best practices. Montreal, Canada: ACI World.

ARMSTRONG, J.S. (2001). "Evaluating forecasting methods". En Armstrong, J.S. (ed.): Principles of Forecasting: A Handbook for Researchers and Practitioners (pp.443-472). 
Norwell, MA: Kluwer Academic Publishers.

BEAULIEU, J.J. y MIRON, J.A. (1993). "Seasonal unit roots in aggregate U.S. data". Journal of Econometrics, 55, pp. 305-328.

BEL, G. y FAGEDA, X. (2011). "La reforma del modelo de gestión de los aeropuertos en España". Hacienda Pública Española / Revista de Economía Pública, 196(1/2011), pp. 109-130.

BOX, G.E. y JENKINS, G.M. (1976). Time Series Analysis: Forecasting and Control. San Francisco: Holden Bay.

BOX, G.E.; JENKINS, G.M. y REINSEL, G.C. (2008). Time Series Analysis: Forecasting and Control. New Jersey: John Wiley \& Sons, Inc.

CARSON, R. T.; CENESIZOGLU, T. y PARKER, R. (2011). "Forecasting (aggregate) demand for US commercial air travel". International Journal of Forecasting, 27(3), pp. 923-941.

COTO-MILLÁN, P. y CARRERA-GÓMEZ, G. (2004). "An air traffic demand forecasting model applied to Santander airport". International Journal of Transport Economics, 31(2), pp. 197-208.

DICKEY, D.A. y FULLER, W.A. (1979). "Distribution of the Estimators for Autoregressive Time Series with a Unit Root". Journal of the American Statistical Association, 74(366), pp. 427-431.

ERICSSON, N.R. (2016). "Economic Forecasting in Theory and Practice: An Interview with David F. Hendry". International Finance Discussion Papers 1184.

GRAEFE, A. (2015). "Improving forecast using equally weighted predictors". Journal of Business Research, 68, pp.1792-1799.

GREEN, K.C. y ARMSTRONG, J.S. (2015). "Simple versus complex forecasting: the evidence". Journal of Business Research, 68, pp.1678-1685.

GUJARATI, D. (2004). Econometría. México D.F.: McGraw-Hill.

HENDRY, D. F. y RICHARD, J.F. (1983). "The Econometric Analysis of Economic Time Series". International Statistical Review, 51(2), pp.111-148.

HYLLEBERG, S.; ENGLE, R.F.; GRANGER, C.W.J. y YOO, B.S. (1990). "Seasonal Integration and Co-integration". Journal of Econometrics, 44, pp. 215-228.

INGLADA, V. y REY, B. (2004). "Spanish air travel and the September 11 terrorist attacks: a note". Journal of Air Transport Management, 10(6), pp. 441-443.

KANG, I. (2003). "Multi-period forecasting using different models for different horizons: an application to U.S. economic time series data". International Journal of Forecasting, 19(3), pp. 387-400.

KLEIN, L.R. (1969). "The Specification of Regional Econometric Models". Papers in Regional Science, 23(1), pp. 105-116.

KLEIN, L.R. y KUSHNIRSKY, F.I. (2005). "Econometric modeling at mixed frequencies". Journal of Economic \& Social Measurement, 30(4), pp. 251-277.

KLEINHENZ, J. (2016). "The art and science of economic forecasting". https://nrf.com/news/the-art-and-science-of-economic-forecasting. [Último acceso: abril de 2017].

LIM, C. y MCALEER, M. (2002). "Time series forecasts of international travel demand for Australia”. Tourism Management, 23, pp. 389-396. 
LÓPEZ, A.M. (2015). "Modelización econométrica regional en España: una revisión aplicada del enfoque unirregional y multirregional". Estudios de Economía Aplicada, 33(2), pp. 399-420.

LÓPEZ, A.M. (2016a). "Applied Economics and Econometrics: Models and Accounting Systems, the origin and future trends". En Hyeronimi, O. y Lo Cascio, M. (eds.): New social market economy for the 21st Century. Emilio Fontela: Economist and global researcher (pp. 179-188). Roma: Aracne Editrice.

LÓPEZ, A.M. (2016b). "El papel de la información económica como generador de conocimiento en el proceso de predicción: Comparaciones empíricas del crecimiento del PIB regional". Estudios de Economía Aplicada, 34(3), pp. 553-582.

MIN, J.C.; KUNG, H.-H. y LIU, H.H. (2010). "Interventions affecting air transport passenger demand in Taiwan". African Journal of Business Management, 4(10), pp. 2121-2131.

NASIRU, S.; LUGUTERAH, A. y ANZAGRA, L. (2013). "The Efficacy of ARIMAX and SARIMA Models in Predicting Monthly Currency in Circulation in Ghana". Mathematical Theory and Modeling, 3(5), pp. 73-81.

PRIYADARSHANA, M. y SHAMINI, A. (2015). "Modeling air passenger demand in Bandaranaike International Airport, Sri Lanka". Journal of Business \& Economic Policy, 2(4), pp. 147-151.

PULIDO, A. (2006). "La aventura de la predicción económica y empresarial". En Pulido, A. (coord.): Guía para usuarios de predicciones económicas (pp. 9-30). Madrid: Ecobook.

PULIDO, A. (2007). "Predicción económica: el reto de la globalización". En Cabañes, L. (coord..): Abstracción y realidad en la economía, ensayos en homenaje al profesor Alejandro Lorca Corrons (pp. 373-377). Madrid: Minerva.

PULIDO, A. (2016). "Mosaico de predicciones para el debate a cuatro". En http://blog.antoniopulido.es/mosaico-de-predicciones-para-el-debate-a-cuatro. [Último acceso: abril de 2017].

PULIDO, A. y LÓPEZ, A.M. (1999). Predicción y simulación aplicada a la economía y gestión de empresas. Madrid: Ediciones Pirámide.

THEIL, H. (1966). Applied economic forecasting. Amsterdam: North Holland.

TSUI, W.H.K. y BALLI, F. (2017). "International arrivals forecasting for Australian airports and the impact of tourism marketing expenditure". Tourism economics: the business and finance of tourism and recreation, 23, pp. 403-428.

TSUI, W.H.K.; BALLI, H.O.; GILBEY, A. y GOW, H. (2014). "Forecasting of Hong Kong airport's passenger throughput". Tourism Management, 42, pp. 62-76.

UNWTO (2013). Notas metodológicas de las bases de datos de estadísticas de turismo. Madrid: United Nations World Tourism Organization.

UNWTO (2016). UNWTO Tourism Highlights, 2016 Edition. Madrid: United Nations World Tourism Organization.

VELASCO, R. (2016). Economistas. Oficio de profetas. Madrid: Catarata.

WEF (2017). The Travel \& Tourism Competitiveness Report 2017, World Economic Forum. Geneva.

WILLIAMS, B.M. (2007). "Multivariate vehicular traffic flow prediction: evaluation of ARIMAX modelling". Transportation Research Board, 1776, pp. 194-200. 